\title{
Micro-Raman spectroscopy for assessment of periodontal disease follow-up
}

\section{Carlo Camerlingo $^{1 \%}$, Fabrizia d'Apuzzo ${ }^{2}$, Vincenzo Grassia ${ }^{2}$, Letizia Perillo ${ }^{2}$, Maria Lepore ${ }^{3}$}

1 CNR-SPIN, Istituto superconduttori, materiali innovativi e dispositivi, via Campi Flegrei 34, 80078 Pozzuoli, Italy; E-Mail:carlo.camerlingo@spin.cnr.it

2 Dipt. multidisc. Spec.medico-chirurgiche e odontoiatriche, Seconda Università di Napoli, Via L. de Crecchio 6, 80138 Napoli, Italy; E-Mails: fabriziadapuzzo@gmail.com (F. d'A.); grassiavincenzo@ libero.it (V. G.); letizia.perillo@unina2.it (L. P.)

3 Dipt. di Medicina Sperimentale, Seconda Università di Napoli, Via S. Maria di Costantinopoli 16, 80138 Napoli, Italy; E-Mail: maria.lepore@unina2.it

* Author to whom correspondence should be addressed; E-Mail: carlo.camerlingo@ spin.cnr.it; Tel.: +39-081-867-5044; Fax: +39-081-804-2519.

Published: 1 June 2014

\begin{abstract}
Micro-Raman spectroscopy is a very promising tool for medical applications, thanks to its sensitivity to subtle changes in the chemical and structural characteristics of biological specimens. We investigated the potentiality of this technique for a follow-up of periodontal disease by analyzing samples of gingival crevicular fluid obtained in not invasive way from informed patients affected by periodontal disease and health ones.

The crevicular fluid was collected on absorbent filter paper cones by putting them inside the tooth sulcus. The fluid impregnated cones were tested by micro-Raman spectroscopy in order to point out differences between samples obtained by disease and health patients. The presence of an excess of carotene is evinced in the spectra of samples from disease affected patients
\end{abstract}

Keywords: Micro-Raman spectroscopy; wavelet data analysis ; periodontitis; gingival crevicular fluid GCF; 


\section{Introduction}

Periodontitis disease is widely diffused in adults in a greater or lesser degree. An early diagnostics is important in order to prevent chronic inflammations and serious oral illness. Visual inspection and radiography of oral cavity are unlikely able to evince early stage of periodontitis. For this reason methods for sensing biomarkers of illness state have been investigated consisting in analyzing saliva [1] or gingival crevicular fluid (GCF) [2]. The periodontal disease is triggered by pathogens substances that stimulate the local synthesis of host immune products [3]. This substances together with products from degraded connective tissue are argued to be present in the saliva and GCF. Indeed, an increasing trend of osteopontin level in GCF from health to gingivitis to periodontitis has been reported in Ref. 2. Micro-Raman Spectrometry ( $\mu$-RS) is an efficient method for characterize biological specimens thanks to its sensitivity to subtle changes in the chemical and structural characteristics $[1,4]$. We investigated the potentiality of this technique for follow up periodontal disease by analyzing samples of GCF obtained in not invasive way from informed patients affected by periodontal disease and health ones. Sterile paper cones imbued by GCF were analyzed by $\mu$-RS. In order to point out the Raman signal of GCF and subtract the contribution due to the paper substrate a suitable numerical treatment of data has been performed and described in detail in the experimental section.

\section{Experimental Section}

\subsection{Sample preparation}

The $\mu$-RS was performed on GCF samples. The fluid was pooled from informed periodontal and health patients by using standardized sterile absorbent paper cones inserted $1 \mathrm{~mm}$ into the gingival crevice and left in situ for $30 \mathrm{~s}$, without blood, saliva and plaque contamination (see Fig.1).

Figure 1. (a) Schematic view of GCF collection method.

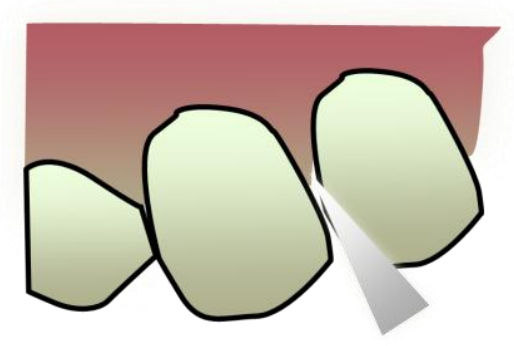

\subsection{Micro-Raman spectrometry}

The GCF sample was excited by the light of a He-Ne laser operating at a wavelength $\lambda=633 \mathrm{~nm}$, with a maximum nominal power of $17 \mathrm{~mW}$. The signal was collected by a Jobin-Yvon TriAx 180 monocromator, equipped with a liquid $\mathrm{N}_{2}$ cooled CCD and a grating of 1800 grooves $/ \mathrm{mm}$, allowing a spectral resolution of $4 \mathrm{~cm}^{-1}$. The laser light is focused on the sample surface by means of a $100 \mathrm{X}$ (n.a.=0.90) optical objective on a excitation area of about $10 \mu \mathrm{m}$ of size. The spectra were obtained using accumulation times ranging in 60-300 seconds. 


\subsection{Wavelet based numerical data treatment}

The spectra typically show a smeared background signal, with intensity of order of about $80 \%$ of the whole average intensity. In order to enhance signal readability and attenuate background and noise components we use a numerical data treatment based on wavelet algorithm [5]. The spectrum signal is cut up into different 'scale' components, by using spatially localized functions with average zero value (namely wavelets, small waves) instead of conventional Fourier transform sinusoidal functions. This make possible to keep information on both frequency and spatial dependence of the signal. Basically the signal is represented in terms of the sum of elementary wavelets and decomposed in two signals, one containing the low frequency components (approximation A) and the other one the fluctuations (detail D). The algorithm is iteratively applied to the "approximated" part of the function and a higher level of A and D component pair is generated. A hierarchical representation of the data set is thus obtained allowing a multi-resolution analysis, know as discrete wavelet transform (DWT). Starting from the decomposed signal, the spectrum can be reconstructed (IDWT) removing low and high frequency components due to spurious background and non-correlated noise signal respectively. MATLAB 6.5 program (by MathWorks Inc.) was used for wavelet analysis with wavelet family of biorthogonal functions "bior6.8". For signal interpretation the spectra were analyzed in terms of convoluted Lorentzian shaped vibration modes. Peaks constituting the spectrum were manually selected in order to define the starting conditions for a best-fit procedure based on the LevenbergMarquardt nonlinear least-square method to determine convolution peaks with optimized intensity, position and width.

\section{Results and Discussion}

The Raman spectrum reported in Fig. 2 ( $a$ and $b$ ) was obtained from the measure of a GCF sample taken from a health patient. The substrate contribution and background signals have been subtracted with the data treatment before described. The spectrum is characterized by typical vibrational modes of biological tissues and proteins. Peaks assigned to Amide III $\left(1240-1300 \mathrm{~cm}^{-1}\right), \mathrm{CH}$ deformation modes (1440-1450 $\mathrm{cm}^{-1}$ ) and Amide I (1600-1700 $\mathrm{cm}^{-1}$ ) are clearly discernables (Fig. 2a). At higher wavenumbers the lipid and protein $\mathrm{CH}_{3}$ bond stretching modes are observed (Fig. 2b). When the spectrum is deconvoluted in Lorentzian peaks, the configuration obtained is close to that expected in undamaged organic tissues [4]. The secondary protein structures are clearly evinced in the Amide I region (Fig. 2c) with the predominant peak of the $\alpha$-helix mode at $1655 \mathrm{~cm}^{-1}$. The spectrum region reported in Fig. 2d, also well reproduce the typical configuration observed in health tissues. The spectrum of GCF from a chronic periodontitis affected patient reveals some differences in the secondary protein structure. The spectrum deconvolution in the amide I region is reported in Fig. 3. When compared with Fig 2c, we notice an intense peak at about $1537 \mathrm{~cm}^{-1}$ which is likely due to the formation of isomerization products containing $\mathrm{C}=\mathrm{C}$ groups related to an increase of degraded carotene in GCF [6]. Carotenoid concentration in GCF is actually expected to increase with the severity increase of the disease and in chronic periodontitis, with respect to healthy or gingivitis control [1,7]. Because of the protein nature of the osteopontin, its presence, even if expected, is more difficult to evince by $\mu$-RS and distinguish its contribution from that of the other protein components . 
Figure 2. Raman spectrum of GCF from healthy patient for the $1200-2000 \mathrm{~cm}^{-1}$ (a) and 2500-3100 $\mathrm{cm}^{-1}$ wavenumber range (b). Deconvolution in Lorentzian peaks of the Amide I spectrum region $\left(1500-1750 \mathrm{~cm}^{-1}\right)(\mathbf{c})$ and $\mathrm{CH}_{3}$ region $\left(2800-3000 \mathrm{~cm}^{-1}\right)$ (d).

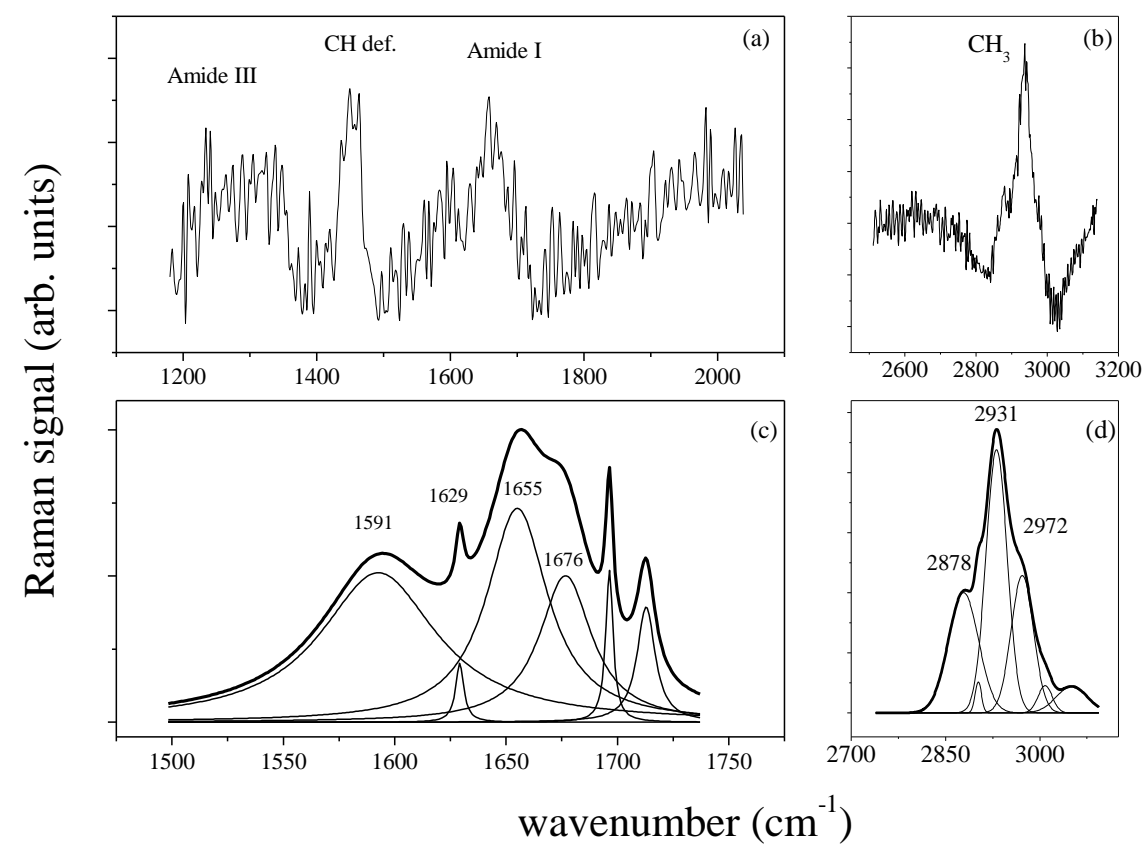

Figure 3. Raman spectrum of GCF from a chronic periodontitis patient: Deconvolution in Lorentzian peaks of the Amide I spectrum region (1500-1750 $\left.\mathrm{cm}^{-1}\right)$.

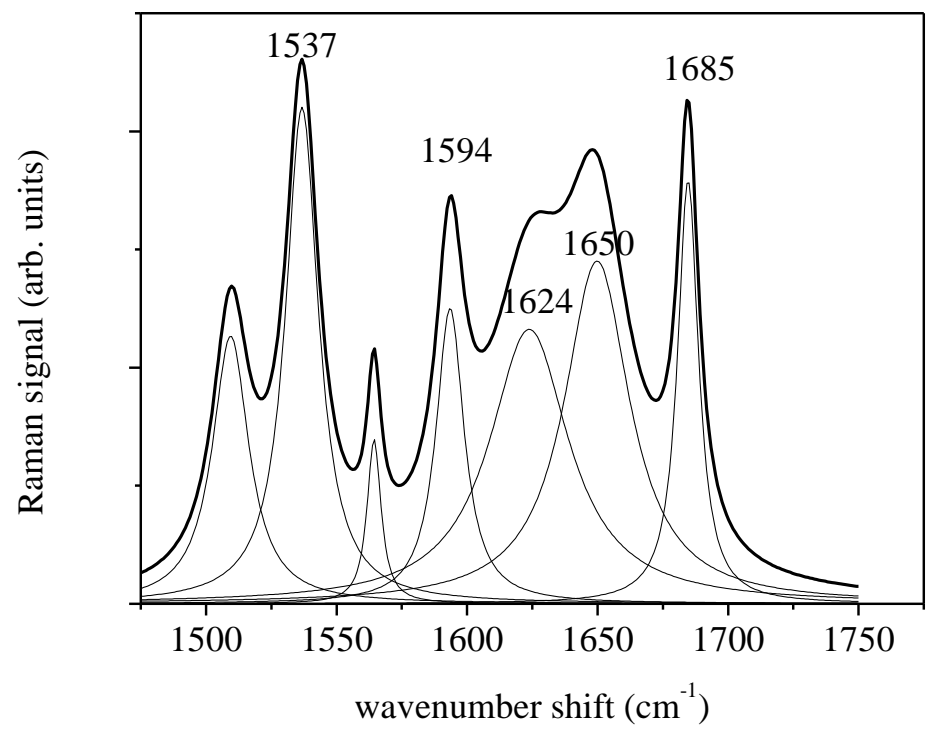




\section{Conclusions/Outlook}

A not invasive method based on $\mu-\mathrm{RS}$ of GCF for follow up of periodontal diseases is proposed and tested. An automatic numerical data treatment based on wavelet algorithm was used in order to suppress the uncorrelated signal, to subtract the background signal and to increase the quantitative readability of the Raman signal. An increase of degraded carotenoid content in GCF, as a mark of the inflammatory state, is inferred from the Raman spectra of chronic periodontit is affected patients.

Even if a more systematic and wide investigation is necessary to validate the proposed methods, the preliminary results confirm the potentiality of $\mu$-RS for specific molecular fingerprinting in medical applications.

\section{Conflicts of Interest}

The authors declare no conflict of interest.

\section{References and Notes}

1. Gonchukov, S.; Sukhinina A.; Bakhmutov D; Minaeva S. Raman Spectroscopy of saliva as perspective method for periodontitis diagnostics. Laser Phys. Lett. 2012, 9,73-77.

2. Sharma, C.G.; Pradeep A.R. Plasma and crevicular fluid osteopontin levels in periodontal health and disease. J. Periodont. Res. 2007, 42, 450-455.

3. d’Apuzzo, F.; Cappabianca, S., Ciavarella, D.; Monsurrò, A.; Silvestrini-Biavati, A.; Perillo, L.; Biomarkers of periodontal tissue remodelling during orthodontic tooth movement in mice and men: Overview and clinical relevance. Scien. World J. 2013, 105873.

4. Camerlingo, C.; Zenone F.; Perna G.; Capozzi V.; Cirillo N.; Gaeta G. M.; Lepore M. An Investigation on Micro-Raman Spectra and Wavelet Data: Analysis for Pemphigus Vulgaris Follow-up Monitoring. Sensors 2008, 8, 3656-3664.

5. Camerlingo, C.; Zenone F.; Gaeta G.M.; Riccio R.; Lepore M. Wavelet data processing of microRaman spectra of biological samples. Meas. Sci. Technol. 2006 , 17, 298-303.

6. Noda, I.; Marcott, C.; Two-dimensional Raman (2D Raman) correlation spectroscopy study of non-oxidative photodegradation of $\beta$-carotene. J. Phys. Chem. A 2002, 106, 3371-3376.

7. Kim, S.C.; Kim, O.-S., Kim, O.-J.; Kim, Y.-J.; Chung, H.-J. Antioxidant profile of whole saliva after scaling and root planing in periodontal disease, J Periodontal Implant Sci 2010, 40, 164-171.

(C) 2014 by the authors; licensee MDPI, Basel, Switzerland. This article is an open access article distributed under the terms and conditions of the Creative Commons Attribution license (http://creativecommons.org/licenses/by/3.0/). 